\title{
A randomized controlled trial of genotype-based Coumadin initiation
}

\author{
James K. Burmester, PhD $D^{1}$, Richard L. Berg, $M S^{2}$, Steven H. Yale, MD ${ }^{3}$, Carla M. Rottscheit, BA ${ }^{2}$, \\ Ingrid E. Glurich, PhD , John R. Schmelzer, PhD, and Michael D. Caldwell, MD, PhD
}

\begin{abstract}
Purpose: A randomized controlled trial was conducted in patients initiating warfarin to determine whether algorithms that incorporate genotypes affecting warfarin metabolism and function, and Vitamin $\mathrm{K}$ metabolism improve prediction of therapeutic warfarin dose and anticoagulation management. Methods: A total of 230 patients were randomized to either a clinical arm where dosing algorithms considered only clinical information or an interventional arm where dosing algorithms used clinical and genotypic variables (CYP2C9, CYP4F2, and $V K O R C 1)$. Subjects in the interventional arm were genotyped within 5 hours, and the initial dose was informed by genotype. Primary endpoints were absolute prediction error relative to therapeutic dose, and time in therapeutic target range during the first 14 days. Secondary endpoints included time to stable dose in therapeutic range, time to first international normalization ratio $>4$, and warfarin-related adverse events. Results: The model including genetics more accurately identified therapeutic dose twice as often as the clinical model $(65.3 \%$ vs. $34.7 \%)$ $(P<0.0001)$. Patients in the interventional arm did not achieve greater time in therapeutic range. Study arms were similar regarding time to international normalization ratio $>4$ and adverse events. Conclusion: Genotype-informed dosing clearly improved prediction of therapeutic dose beyond that available with clinical parameters. Genetic information did not affect time in therapeutic target range during the first 14 days of therapy. Current management practices with the vagaries in dose adjustment after warfarin initiation exert a strong influence on traditional clinical outcomes. Genet Med 2011:13(6):509-518.
\end{abstract}

Key Words: anticoagulation, bleeding, CYP2C9, CYP4F2, INR, VKORC1, warfarin dosing

$U^{\mathrm{n}}$ til very recently, warfarin has been the only US Food and Drug Administration-approved oral anticoagulant and is still the drug of choice for long-term anticoagulation. ${ }^{1}$ However, warfarin ranks among the top 10 drugs causing serious adverse events and emergency room visits. ${ }^{2}$ The safe use of warfarin is hampered by a narrow therapeutic index and substantial interindividual variation in dose requirements. Until an individual's therapeutic dose of warfarin is known, patients on warfarin therapy are at high risk for serious adverse health events,

From the Departments of ${ }^{1}$ Human Genetics, ${ }^{2}$ Biomedical Informatics, and ${ }^{3}$ Clinical Research; ${ }^{4}$ Office of Scientific Writing and Publication, Marshfield Clinic Research Foundation; and ${ }^{5}$ Department of Surgery, Marshfield Clinic, Marshfield, Wisconsin.

Michael D. Caldwell, MD, PhD, Department of Surgery, 1000 North Oak Avenue, Marshfield, WI 54449. E-mail: caldwell.michael@marshfieldclinic.org.

Disclosure: M.D.C., R.L.B., and J.K.B. are coinventors of patent application $12 / 251,540$ and CT/US2008/079966 pending investigation that is property of Marshfield Clinic.

Submitted for publication August 24, 2010.

Accepted for publication December 9, 2010.

Published online ahead of print March 17, 2011

DOI: $10.1097 /$ GIM.0b013e31820ad77d especially during drug initiation and when the international normalization ratio (INR) is above the therapeutic target range. ${ }^{3}$

Interindividual variation in warfarin dose is mediated by multiple factors. ${ }^{4-6}$ Approximately $20 \%$ of the variability is explained by age, presence of comorbidities (e.g., diabetes, cancer, renal or liver disease, and concomitant use of some medications), and other personal characteristics (e.g., gender, smoking, and body size). ${ }^{7}$ An additional $35 \%$ of dose variability is attributed to polymorphisms in CYP2C9, VKORC1, and CYP4F 2 genes. ${ }^{8-10}$ For each of these genes, there is a clear biological pathway linking genetic variations to warfarin dose response. The CYP2C9 enzyme metabolizes warfarin, and polymorphisms in this gene reduce enzymatic activity, leading to markedly lower therapeutic doses of warfarin in patients with these polymorphisms. Warfarin blocks VKORC1 enzyme activity, which catalyzes the reduction of Vitamin $\mathrm{K}_{1}$ and its 2,3 epoxide. The reduced form of these compounds serves as a cofactor for the gamma glutamyl carboxylase that generates the active form of clotting factors II, V, VII, and IX. The genetic variants in VKORC1 result in a reduction of warfarin dose requirement by approximately $25 \%$ per variant allele. ${ }^{9}$ The Cyp4F2 enzyme cleaves the phytal side chain of Vitamin K leading to inactive metabolites. ${ }^{11}$ The genetic polymorphism in CYP4F2 increases warfarin therapeutic dose by up to $12 \%$ per allele. ${ }^{10}$ Advanced models using combinations of clinical attributes and these genetic factors explain $50-55 \%$ of variability in warfarin dose requirements. ${ }^{12,13}$

The development of warfarin dosing models, leveraged significantly by recent genetic discoveries, spurred interest in their potential application to improve patient safety by reducing or eliminating serious adverse health events. Therefore, we conducted a prospective, randomized, blinded, twoarm trial to determine whether initial warfarin dosing based on an algorithm using relevant genetic polymorphisms and clinical parameters (genetic + clinical arm) was superior to an algorithm using only usual clinical parameters (clinicalonly arm) in predicting stable therapeutic dose of warfarin and in anticoagulation outcomes.

\section{METHODS}

\section{Study objective}

The objectives of the study were to determine the degree to which algorithms using clinical and genotypic variables that affect warfarin metabolism and function improve prediction of therapeutic warfarin dose and management of anticoagulation during the initiation period compared with clinical variables alone. Primary study endpoints were (1) absolute prediction error relative to therapeutic dose and (2) time in therapeutic target range during the first 14 days of therapy.

\section{Patient eligibility, consent, and enrollment}

The study was approved by the Institutional Review Board of Marshfield Clinic. Additional oversight was provided by a Data 
Safety Monitoring Board (DSMB), which met three times during the course of the trial. The DSMB reviews focused on safety data, but one meeting included a formal interim analysis. The study was registered at www.clinicaltrials.gov under protocol NCT00484640: Modeling genotype and other factors to enhance safety of coumadin prescribing before enrollment of subjects. All participants provided written informed consent before trial enrollment.

Potentially eligible subjects included those new to warfarin therapy and those restarting therapy without a documented stable warfarin dose available in the medical record. Most subjects were initially identified through screening of the Marshfield Clinic electronic medical record for medical indications that require warfarin therapy. Some patients were referred to the study by physicians at Marshfield Clinic. Additional inclusion criteria were established to ensure that subject characteristics aligned closely with characteristics of the population on which algorithm parameters were estimated, including male and female patients of white ancestry (including Hispanic white), 40 years of age or older, and target INR range between 2 and 3.5. The dosing algorithm was limited to whites during development as the available population was $>98 \%$ white, with insufficient numbers of subjects of other races. The study was initiated before the recent publication of other algorithms and was the only algorithm at the time that included CYP4F2. Exclusion criteria included pregnancy, known Native American, African American or Asian descent, thrombocytopenia, severe to moderate hepatic insufficiency, and other clinical contraindications for warfarin as deemed by the patient's physician. Women of childbearing potential were required to use an effective method of contraception.

\section{Blinding}

Study investigators who performed genetic testing were blinded to all aspects of patient care. Study physicians, nurses, and research coordinators were blinded to randomization of the subjects and to genotype. Patients were not blinded to their initial dose or dose adjustments. Attending physicians had access to all dose data but not to genotype or study arm assignment of their patients.

\section{Study period}

The study was designed to assess the impact of geneticinformed initial warfarin dose on commonly accepted clinical indicators, such as time in therapeutic range within the early initiation period when some experts, including ourselves, anticipated the effects of genotype-based dosing would be most evident. ${ }^{14}$ A 60 -day period was selected as a compromise between the more common 90-day period and a shorter 30-day period, which was thought to capture the effects of genotypebased initial dosing. ${ }^{15}$

\section{Study intervention}

\section{Genetic testing}

Genetic testing was performed in compliance with Clinical Laboratory Improvement Act-regulated procedures. The $C Y P 2 C 9$, $V K O R C 1$, and $C Y P 4 F 2$ genotypes were determined using Invader ${ }^{\circledR}$ chemistry in a laboratory analysis developed with analyte-specific reagents manufactured by Third Wave Technologies (TWT, Madison, WI). The CYP2C9 assay detects $C Y P 2 C 9 * 2$ and $C Y P 2 C 9 * 3$. The VKORC1 assay detects position-1639. The $C Y P 4 F 2$ assay detects rs2108622. The $C Y P 2 C 9$ and $V K O R C 1$ assays have accuracy $>99 \% .^{16,17}$ Before initiation of the trial, the CYP4F2 assay was validated with $100 \%$ concordance by sequencing nine samples using Big Dye Ter- minator 3.1 cycle sequencing with results read on an Applied Biosystems Prism ${ }^{\mathrm{TM}} 3100$ Genetic Analyzer. Twenty-one samples were tested by both the TWT CYP4F2 research-use-only assay and Affymetrix DMET panel with $100 \%$ concordance. Additionally, synthetic targets for both alleles were tested and gave the expected result. Finally, four sets of trios from the HapMap project obtained from Coriell Institute for Medical Research (Camden, NJ) were tested and gave the correct result.

Leukocyte DNA was extracted from blood samples using QIAamp blood mini kit (Qiagen Inc., Valencia, CA). A positive control (DNA sample heterozygous for each allele of each gene tested), a negative control (water), and a heterozygous synthetic target were analyzed with each run to ensure that the test was functional. Test results were copied directly from the TWT report form into a dose calculation application within the study database. Twice per year, samples selected to cover all combinations of alleles were retested using the TWT assay and sequenced using Big Dye terminators to validate accuracy of the assay.

\section{Initial warfarin dose}

Blood was collected from all subjects at the time of enrollment. Subjects randomized to the genetic + clinical arm were genotyped in real time, so that their initial warfarin dose could be informed by their genotypes. Blood from subjects randomized to the clinicalonly arm was frozen at $-80^{\circ} \mathrm{C}$ and genotyped after these subjects completed the trial. Therapeutic warfarin dose was estimated individually for each subject using the algorithms presented in Table 1. Marshfield pharmacogenetic models for

\begin{tabular}{lcc}
\hline Table 1 Algorithms for initial warfarin dose \\
\hline Variables & $\begin{array}{c}\text { Genetic }+ \text { clinical arm } \\
\text { parameter estimates, } \\
\text { CYP2C9*1 genotypes only }\end{array}$ & $\begin{array}{c}\text { Clinical-only arm } \\
\text { parameter estimate, } \\
\text { regression model } \\
\text { for log (dose) }\end{array}$ \\
\hline Intercept & $4.004 \pm 0.177$ & $3.458 \pm 0.243$ \\
CYP2C9 & $-0.186 \pm 0.017$ & - \\
VKORC1 & $-0.340 \pm 0.019$ & - \\
VKORC1 Het & $0.069 \pm 0.025$ & - \\
CYP4F2 & $0.111 \pm 0.018$ & - \\
Male & $0.061 \pm 0.029$ & $0.085 \pm 0.040$ \\
Valve & $0.165 \pm 0.031$ & $-0.633 \pm 0.264$ \\
Age & $-0.009 \pm-0.001$ & $0.258 \pm 0.085$ \\
BSA & $0.320 \pm 0.063$ & $-0.007 \pm 0.004$ \\
Age*Valve & - &
\end{tabular}

Genetic + clinical arm geometric means, non-*1 genotypes

Genotype

Dose $(\mathrm{mg} / \mathrm{wk})$

$* 2 / * 2$

24.9

$* 2 / * 3$

14.8

$* 3 / * 3$

Definition of coding variables: gender: 1 for male and 0 for female; valve replacement indicator: 1 for yes and 0 for no; age in years; BSA, body surface area $\left(\mathrm{m}^{2}\right)$; CYP2C9 code: 1 for $* 1 / * 1,2$ for $* 1 / * 2$, and 3 for $* 1 / * 3$; VKORCl code: 1 for GG, 2 for heterozygote, and 3 for CC; VKORC1 heterozygote indicator: 1 for het and 0 other; and 4F2 genotype: 1 for $C C, 2$ for $C T$, and 3 for $T T$. 
predicting therapeutic warfarin dose were first developed and validated in $2004^{18}$ and have been further refined and tested since that time..$^{5,10,14}$ These multivariable regression models use relevant clinical parameters with or without genotype information. Because the model development cohort included few patients with no wild-type CYP2C9 alleles (*1), multivariate models were not established for those subjects who were $C Y P 2 C 9 * 2 / * 2, C Y P 2 C 9 * 2 / * 3$, or $C Y P 2 C 9 * 3 / * 3$. For those genotypes, the genetic + clinical algorithm used the simple geometric mean doses from subjects in the model development cohort. The estimated dose was prescribed as the initial dose. All clinical data required for the algorithms were entered into the trial database. Once the data for a subject were entered, a database application randomized the subject and alerted laboratory staff as to whether the blood sample should be stored or genotyped immediately (completed within approximately 5 hours). For those genotyped immediately, laboratory staff entered genetic results into the trial database as soon as they were available. After all data required for the algorithm were entered, the database application calculated the assigned dose and notified nursing staff by e-mail. The database application also allowed trial staff to check on the status of patients enrolled at any time.

Nonhospitalized study subjects were supplied with starter kits containing Coumadin ${ }^{\circledR}$ pills in 1,2 , and $5 \mathrm{mg}$ concentrations to take with them after the clinical visit during which they enrolled. By providing various concentrations, subjects could return home while genotyping was performed. Brand name Coumadin ${ }^{\circledR}$ was used throughout the study to eliminate variability that may be due to differences in formulation among warfarin manufacturers.

Once the assigned dose was available, a trial nurse contacted the subject by telephone and provided instructions on the correct pill or combination to be taken. For subjects recruited during hospital stays, trial nurses and physician investigators coordinated Coumadin ${ }^{\circledR}$ therapy through the hospital pharmacy. All outpatient follow-up was conducted by trial nurses during the 60 -day trial period. Coumadin ${ }^{\circledR}$ was supplied to all subjects without cost for the duration of their 60-day enrollment in the trial. Patients did not receive financial remuneration for participating in this study.

\section{Dose adjustment}

Subjects received normal care for dose adjustments from nurses in the Marshfield Clinic Anticoagulation Service (ACS) or from one of two physician study investigators for hospitalized patients, using well-established ACS guidelines. Established in 1999, the ACS is nurse managed, physician led and has a demonstrated record for high-quality care. ${ }^{19} \mathrm{ACS}$ dose guidelines are based on consensus anticoagulation management strategies from the American College of Cardiology and the American Heart Association and are described by Ansell et al. ${ }^{20}$ Figure 1 summarizes dose-adjustment processes in the first 10 days of therapy. INR measurements made by the clinical laboratory were used to inform dose adjustments.

\section{Randomization}

The trial randomization list was created using a random number generator in $\mathrm{SAS}^{\circledR}$ statistical software $\left(\mathrm{SAS}^{\circledR}\right.$ is the registered trademark of SAS Institute Inc. in the United States and other countries; ${ }^{\circledR}$ indicates US registration). Assignments to the two trial arms were blocked to ensure nearly equal numbers in each arm using randomly alternating block sizes of two and four. The randomization list was stored in the trial database application, but the list could only be accessed by the trial programmer. Randomization of individual subjects occurred after consent but before genotyping using the database application.

\section{Study outcomes}

The primary study endpoints were (1) absolute prediction error relative to therapeutic dose and (2) time in therapeutic target range during the first 14 days of therapy. Secondary endpoints were (1) time to therapeutic dose; (2) time to first INR $>4$; and (3) warfarin-related adverse health events.

\section{Therapeutic dose}

Therapeutic dose for the trial was defined as the average daily dose of warfarin in milligrams required to maintain a patient within target INR range as validated by two in-range measures taken at least 3 days apart, where the dosing regimen was consistent in the period from 6 days before the first INR through the last INR. This definition requires a consistent dosing regimen for at least 10 days, where consistent dosing was defined as requiring that the dose change by no more than $0.5 \mathrm{mg}$ during the period. Although clinical management evaluates dosing on a weekly basis allowing for some variation in dosing from day to day, for trial purposes the total weekly dose was to be administered in seven approximately equal amounts.

For subjects who did not reach a trial-defined therapeutic dose during their trial period $(n=77)$, we examined INR and dose data for an additional 60 days after their trial period to determine whether a clinically defined therapeutic dose could be determined. We developed an estimate of therapeutic dose in a manner analogous to our trial-defined stable dose: two consecutive in-range INRs were required that were at least 3 days apart, and the weekly dose remained constant for the period beginning 6 days before the index INR through the date of the trailing INR. The principal difference between trial and clinically defined dose was that the time period between INR measures tended to be longer in the maintenance phase compared with the initiation period. Ultimately, we were able to determine a clinical therapeutic dose for 23 of the 77 (30\%) subjects who did not achieve therapeutic dose in the trial period.

\section{Dose prediction errors}

To calculate absolute prediction errors relative to therapeutic dose for each subject with data available, we determined the absolute value of the difference between the observed therapeutic dose and the algorithm-estimated dose. The absolute value was used as the endpoint, as both underdosing and overdosing increase patient risk. Because genetic data were available for analyses for all subjects regardless of their randomized assignment, and because the clinical factors were identical in both models, analyses were conducted that compared therapeutic dose with predicted starting doses for both clinical-only and genetics-clinical algorithms in all subjects regardless of randomized assignment. We also evaluated a "standard of care" 5 mg starting dose.

\section{Time in therapeutic target range}

Time in therapeutic target range during the first 14 days was calculated using two methods. The first was the simple percentage of days where INR was within range among those days where INR was measured. The second was as a weighted measure that interpolated the INR between two consecutive tests $^{21}$ and then weighted proportionately by how close the INR was to target, resulting in a risk-weighted outcome measure. 


\section{INITIATION OF WARFARIN THERAPY}

Day $3-4$

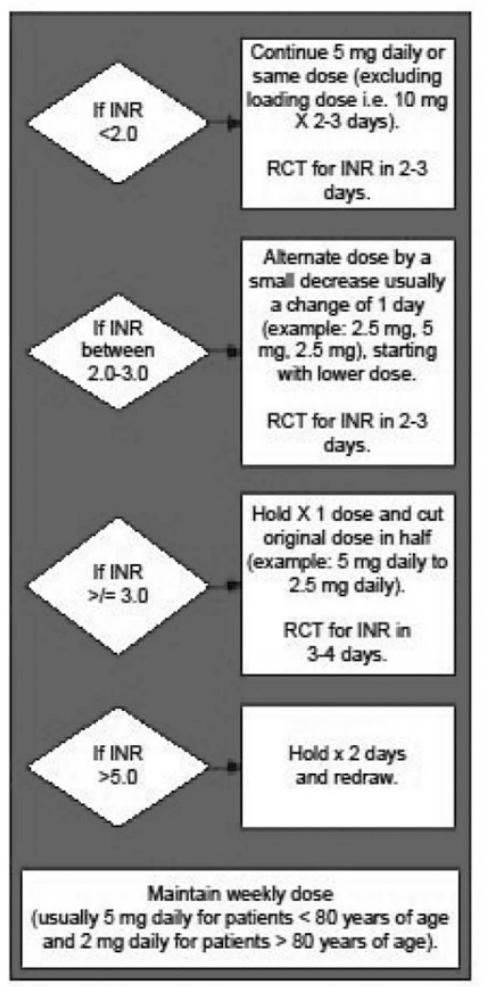

Day $5-7$

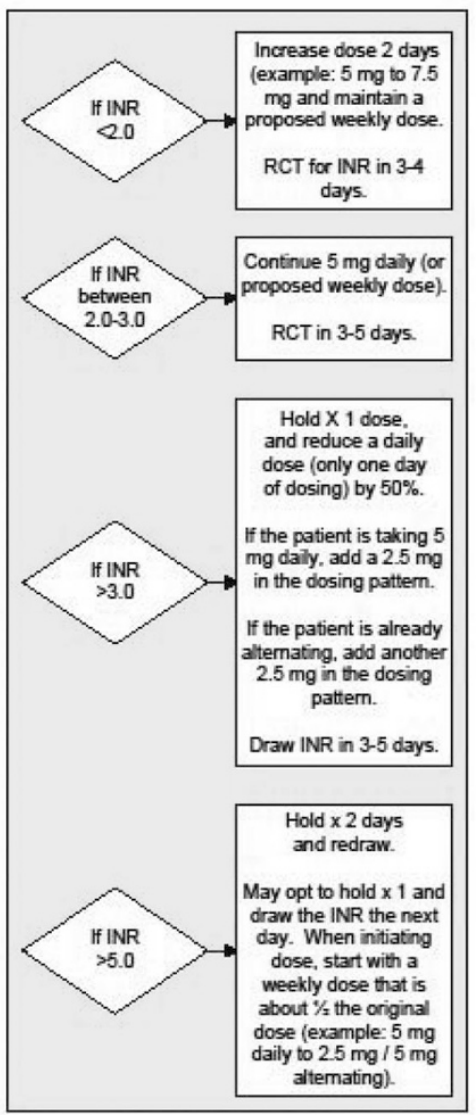

Day 7-10

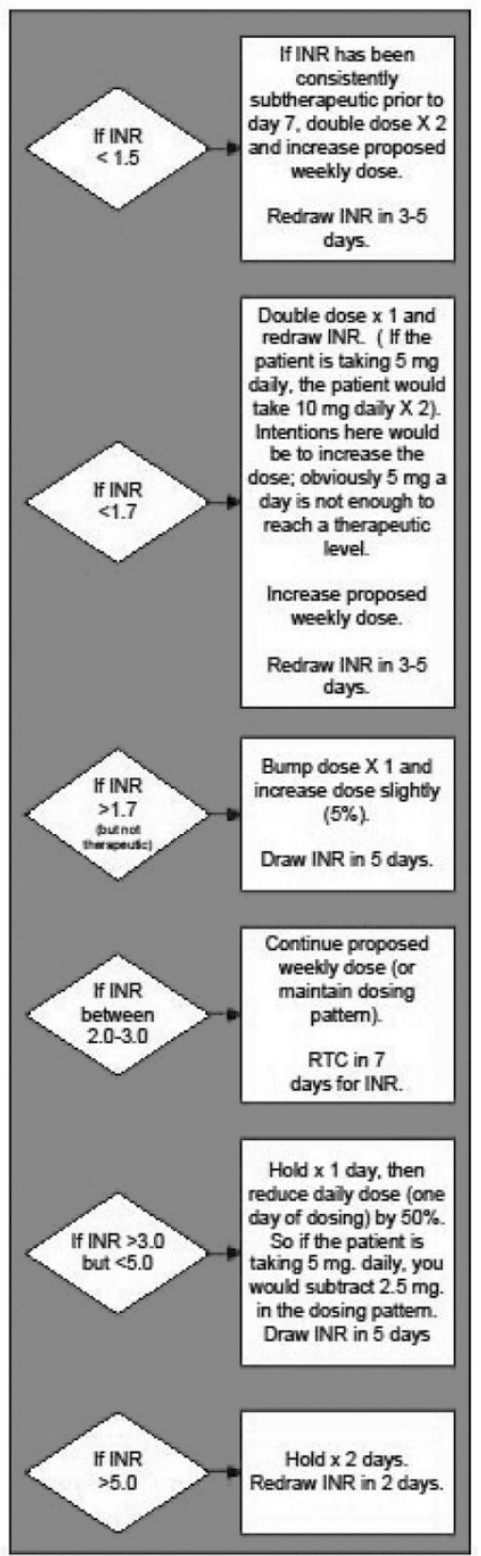

Past Day 10

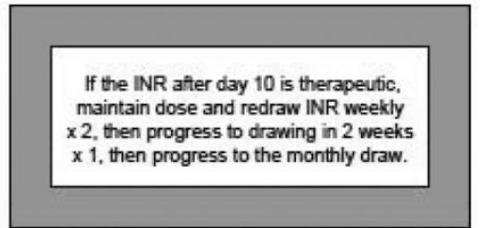

Fig. 1. Dosing decision model during warfarin initiation days 1-10. The dosing decision tree used during the first 10 days after warfarin initiation is shown, which was used to adjust patient warfarin dosing. RCT, refer for clinical testing. 
Table 2 Analysis of time in range for the first 14 study days

INR \% time in range based on the simple fraction

\begin{tabular}{lcccccc} 
& $N$ & Mean & SD & $25 \%$ & Median & $75 \%$ \\
\hline Clinical only & 112 & 30.8 & 18.4 & 14.3 & 28.6 & 42.9 \\
Genetic + clinical & 113 & 29.1 & 15.5 & 16.7 & 28.6 & 37.5
\end{tabular}

$$
p=0.564
$$

INR risk-weighted time in range ${ }^{a}$

\begin{tabular}{lccccrr} 
& $N$ & Mean & SD & $25 \%$ & Median & $75 \%$ \\
\hline Clinical only & 112 & 1.20 & 0.15 & 1.14 & 1.18 & 1.24 \\
Genetic + clinical & 113 & 1.21 & 0.12 & 1.15 & 1.18 & 1.24 \\
& & $p=0.891$ & & & & \\
\hline
\end{tabular}

${ }^{a}$ Weights: 1.25 , INR below range; 1.0 , INR in range; 1.25 , INR near above; 2.0 , INR $>4 ; 3.0$, INR $>6$; and 5.0, INR $>8$.

$\mathrm{SD}$, standard deviation.

The weights were based on published relative risks ${ }^{3,22}$ and the clinical judgment of the study investigators (Table 2).

\section{Time to achieve therapeutic dose}

Time to achieve therapeutic warfarin dose was defined as the number of days from initiation to the first INR used (as above) to determine therapeutic dose.

\section{Time to INR $>4$}

Time to first INR above 4 was measured as the number of days from warfarin initiation to the first reported INR $>4.0$.

\section{Warfarin-related adverse events}

Warfarin-related adverse drug events reported to the DSMB were defined as serious events that occurred during the trial including all deaths and other unanticipated health events, particularly thromboembolic events and serious hemorrhagic events. For completeness, all less serious thromboembolic and hemorrhagic events were also recorded. A standard adverse event report form was used to collect data on the patients as the adverse events occurred. Adverse events were collected through contact with the patients during the study and by abstraction from the patient's electronic medical record.

\section{Statistical power and sample size}

The trial recruitment goal was 130 subjects in each arm. Based on our data from previous studies, this was estimated to provide at least $80 \%$ statistical power (two-sided tests with $\alpha=$ 0.05 ) for each of our two primary outcomes assuming effect sizes of $15 \%$ and evaluable data on at least $90 \%$ of those enrolled.

One formal interim analysis was planned using an O'Brien and Fleming ${ }^{23}$ bound $(\alpha=0.005$ for the interim analysis, and $\alpha=0.048$ for the final analyses). Evaluation of the interim analysis resulted in recommendation by the DSMB for continuation of the trial.

The rate of enrollment was slower than anticipated, and even though trial enrollment was extended from 12 to 19 months, final enrollment achieved was 115 subjects per arm $(88.5 \%$ of goal). After conditional power calculations showed very low power for observing a significant difference with respect to time in therapeutic range, even if the targeted enrollment was pursued, the trial investigators, the trial sponsor (Agency for
HealthCare Research and Quality), and the DSMB agreed that the trial should close.

\section{Statistical analysis}

Baseline characteristics were summarized by randomized arm to describe the subjects enrolled and to illustrate the balance achieved by the randomization. The simple and riskweighted time-in-range variables were summarized with medians, and the trial arms were compared using the Wilcoxon rank-sum test. The primary analysis of the absolute prediction errors was based on a general linear mixed model. Our previous experience with this outcome showed a distribution that was skewed but was well normalized by Box-Cox ${ }^{24}$ transformations. The primary analyses were, therefore, performed on the optimally transformed measures. The errors under each model were calculated for each patient, one for each study arm, with the correlation of these repeated measures within subjects incorporated in the mixed model. An additional term was added to the model to indicate the trial arm actually assigned and to allow the possibility that the initial dose assigned somehow affected the determination of therapeutic dose.

Secondary analyses included time-to-event (survival) analyses for the time to achieve therapeutic dose and for the time to the first INR $>4$. The Kaplan-Meier method $^{25}$ was used to estimate the survival functions, and tests of differences in eventfree survival were based on the generalized Wilcoxon test. ${ }^{26}$

It was expected that serious adverse events among participants during the trial would be rare and generally unrelated to study assignment. Given the low statistical power for event comparisons, adverse events were described by trial arm but were not statistically compared by arm.

\section{RESULTS}

Study enrollment occurred over a 19-month period, beginning in June 2007, during which 1971 patients were screened for eligibility, and 230 were enrolled and followed throughout the trial as shown in Figure 2. Only $20(1 \%)$ eligible patients declined enrollment into the study. The primary reasons for screen failure were (1) a previously documented warfarin dose history, (2) physician treatment decisions, (3) logistic challenges (e.g., patient was a nursing home resident), and (4) inadequate time to enroll and genotype prospective subjects 


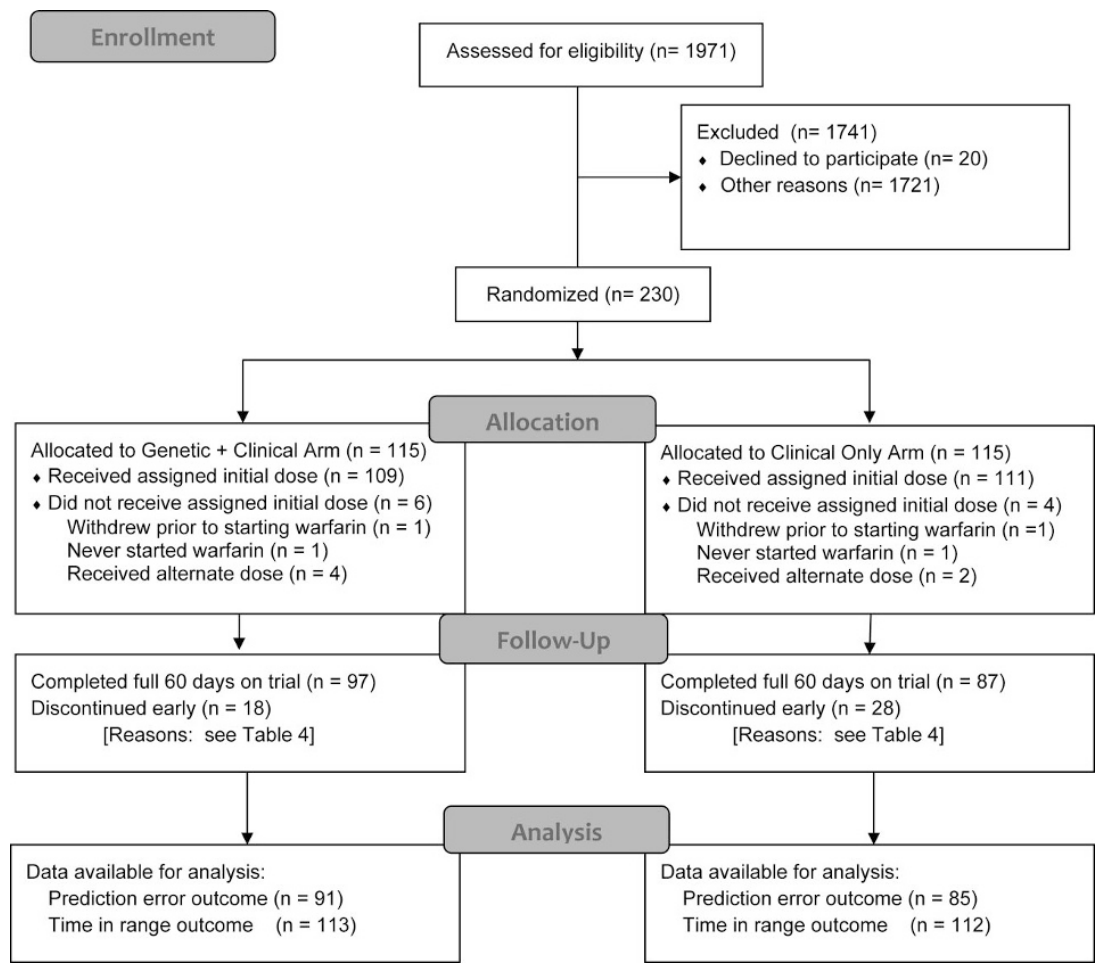

Fig. 2. Flow diagram showing the number of trial subjects at each stage.

before their first warfarin dose due to either late identification of potential subjects or late decisions by physicians to initiate the patient on warfarin.

Study subject demographics by study arm are summarized in Table 3. The arms were similar at randomization with respect to gender, age, body surface area, and indication for anticoagulation therapy. Genotypes by study arm are summarized in Table 4. Although the frequency of the VKORC1 " $G / G$ " allele was somewhat higher in the genotype + clinical arm (46\% vs. $36 \%)$, the distributions were not significantly different $(P=0.305)$.

The final study status by randomized arm for all 230 subjects is summarized in Figure 2 and Table 5. A total of five withdrew consent, including two who withdrew before starting warfarin. In addition, two other subjects enrolled but did not start warfarin therapy. Four patients died while in active follow-up (none due to trial-related causes). Thirty-five subjects discontinued early (including one who subsequently died), with the primary reason for discontinuation being the termination of warfarin therapy. One hundred eighty-four subjects $(80 \%)$ completed the entire 60 -day trial period, with a total of 12,104 subject-days accrued in the trial. Even among those who did not complete the entire trial, a large majority (42/46) provided some data for analyses before they discontinued. Therapeutic dose was established for 176 subjects, 91 of $115(79 \%)$ in the genetic + clinical arm and 85 of $115(74 \%)$ in the clinical-only arm.

There were 65 dosing deviations documented during the study (Table 6). Six subjects inadvertently received their first dose based on physician orders rather than trial assignment. This occurred twice when hospital staff started a subject on warfarin before notifying study staff, twice due to errors by trial staff, once due to a genotyping delay, and once due to a database error. In these cases, the assigned trial dose was started
Table 3 Demographics of randomized arms

\begin{tabular}{lcc}
\hline & Clinical only & Genetic + clinical \\
\hline$N$ & 115 & 115 \\
$\%$ Female & 39 & 43 \\
Indication for warfarin & & \\
$\quad$ Arrhythmia & $49 \%$ & $43 \%$ \\
$\quad$ Thromboembolic disease & $38 \%$ & $38 \%$ \\
$\quad$ Valve surgery & $17 \%$ & $21 \%$ \\
Hospitalized at initiation & $81 \%$ & $80 \%$ \\
Days hospitalized after initiation & & \\
$\quad$ Median (min, max) & $3(1,85)$ & $3(1,33)$ \\
Age (yr) & & \\
$\quad$ Median (min, max) & $69.2(40.0,90.6)$ & $67.4(40.4,89.6)$ \\
Body surface area & & \\
$\quad$ Median (min, max) & $1.98(1.23,2.95)$ & $1.96(1.36,2.65)$ \\
\hline
\end{tabular}

on Day 2 of therapy. The overwhelming majority of dosing deviations were documented events of subjects forgetting to take a dose or instances where a nontrial physician treated the these deviations are similar to what is observed in the usual clinical management of warfarin patients.

Dose changes within the first 3 days of therapy were attributable to changing patient conditions, including indica- 
Table 4 Genotype by randomized arm

\begin{tabular}{ccc}
\hline & \multicolumn{2}{c}{$N(\%)$} \\
\cline { 2 - 3 } Clinical only & Genetic + clinical \\
$* 1 / * 1$ & $67(59.8)$ & $73(64.0)$ \\
$* 1 / * 2$ & $26(23.2)$ & $25(21.9)$ \\
$* 1 / * 3$ & $16(14.3)$ & $12(10.5)$ \\
$* 2 / * 2$ & $1(0.9)$ & $2(1.8)$ \\
$* 2 / * 3$ & $2(1.8)$ & $1(0.9)$ \\
$* 3 / * 3$ & 0 & $1(0.9)$ \\
$V K O R C 1$ & & $52(45.6)$ \\
$G G$ & $40(35.7)$ & $43(37.7)$ \\
$G A$ & $52(46.4)$ & $19(16.7)$ \\
$A A$ & $20(17.9)$ & \\
$C Y P 4 F 2$ & & $59(51.8)$ \\
$C C$ & $53(47.3)$ & $51(44.7)$ \\
$C T$ & $49(43.8)$ & $4(3.5)$ \\
$T T$ & $10(8.9)$ &
\end{tabular}

Table 5 Final subject status

\begin{tabular}{|c|c|c|c|c|}
\hline & \multicolumn{4}{|c|}{ Randomized arm } \\
\hline & \multicolumn{2}{|r|}{$\begin{array}{l}\text { Clinical } \\
\text { only }\end{array}$} & \multicolumn{2}{|c|}{$\begin{array}{l}\text { Genetic + } \\
\text { clinical }\end{array}$} \\
\hline & $N$ & Percentage & $N$ & Percentage \\
\hline Completed 60 days & 87 & 75.7 & 97 & 84.3 \\
\hline $\begin{array}{l}\text { Discontinued protocol early- } \\
\text { deceased }\end{array}$ & 3 & 2.6 & 1 & 0.9 \\
\hline $\begin{array}{l}\text { Discontinued protocol early- } \\
\text { may follow }{ }^{a}\end{array}$ & 20 & 17.4 & 15 & 13.0 \\
\hline $\begin{array}{l}\text { Discontinued protocol early- } \\
\text { withdrew }\end{array}$ & 3 & 2.6 & 0 & 0.0 \\
\hline $\begin{array}{l}\text { Enrolled but never started } \\
\text { warfarin }\end{array}$ & 1 & 0.9 & 1 & 0.9 \\
\hline $\begin{array}{l}\text { Enrolled withdrew before } \\
\text { initiation }\end{array}$ & 1 & 0.9 & 1 & 0.9 \\
\hline
\end{tabular}

tions for warfarin, surgery/procedures, and bleeding ( $n=$ $11)$; unanticipated steep increases in INR $(n=27)$ often accompanied by other drug initiation (e.g., Amiodarone, Levaquin, or statins) or low INR response $(n=5)$; dose inconsistency in hospital records $(n=2)$; late study enrollment $(n=1)$; or patient error $(n=2)$.

\section{Prediction errors}

Actual and predicted doses for the 176 study subjects (76\%) with therapeutic dose determined are shown in Figure 3. Sub-
Table 6 Frequency of dose deviations

\begin{tabular}{lccc}
\hline & \multicolumn{3}{c}{ Randomized arm } \\
\cline { 2 - 4 } & Clinical only & Genetic + clinical & Combined \\
\hline $\begin{array}{l}\text { Deviation from } \\
\text { assigned initial } \\
\text { dose }\end{array}$ & 2 & 4 & 6 \\
$\begin{array}{l}\text { Other dosing } \\
\text { deviation }\end{array}$ & 35 & 25 & 60 \\
$\begin{array}{l}\text { Combined } \\
\text { Oom }\end{array}$ & 37 & 28 & 65 \\
\hline May include multiple actions per subject. & & \\
\hline
\end{tabular}

jects in the figure are sorted from left to right in order of the relative errors in the two models. The 61 subjects $(34.7 \%)$ on the left side of Figure 3 were better predicted by the clinicalonly model, while the 115 subjects $(65.3 \%)$ on the right were better predicted by the genetic + clinical model. The model including genetics provided the best prediction of therapeutic dose nearly twice as often as the clinical model. The median absolute prediction error was $1.32 \mathrm{mg}$ /day for the clinical-only model, and $0.80 \mathrm{mg} /$ day for the genetic + clinical model. The median difference in absolute errors between models was 0.39 $\mathrm{mg}$ /day ( $95 \%$ confidence limits: $0.26-0.57 \mathrm{mg} /$ day), favoring the model which included genotype.

The results provide very strong evidence that the model including genotype predicts therapeutic dose better than the clinical-only model $(P<0.0001)$. Moreover, both trial models were superior to a common clinical "standard of care" of 5 $\mathrm{mg} /$ day in predicting therapeutic dose. Predictions from the genetic + clinical model were better in $69 \%$ of comparisons with $5 \mathrm{mg} /$ day dosing, whereas the clinical-only model was better in $60 \%$ of comparisons.

\section{Time in therapeutic target range}

Summaries of the primary study endpoint of time in range are presented in Table 6. A total of 225 (98\%) subjects had some INR results in the first 14 days after enrollment for analysis, with between 1 and 14 INR results (median 8 in each arm) available per subject. The medians for the simple percent time in range were $28.6 \%$ in both arms $(P=0.564)$. The riskweighted time in range has been scaled (Table 6) relative to target, with the ideal INR (minimum risk) scaled as 1.0, and the value increasing proportionally from 1.0 for INRs further from the target, to a maximum risk of 1.25 for INRs below range, and to a maximum risk of 5.0 for INRs $>8$. The medians for risk-weighted time in range were 1.18 in both arms with no evidence of differences in time in range between the randomized arms $(P=0.891)$.

\section{Time to stable therapeutic dose}

Observed times to stable therapeutic dose were very similar in the two arms $(P=0.90)$. Estimates of the median times to stable therapeutic dose were 31 days (95\% confidence interval: $24-36$ days) in the clinical-only arm and 29 days (95\% confidence interval: 23-36 days) in the genetic + clinical arm. Therapeutic dose was established in 176 subjects. Subjects for whom therapeutic dose could not be reliably determined $(n=$ 54) included subjects who withdrew from the trial, died, or were taken off warfarin for clinical reasons, as well as some where the therapeutic dose was difficult to establish and was not determined by the end of the trial. 


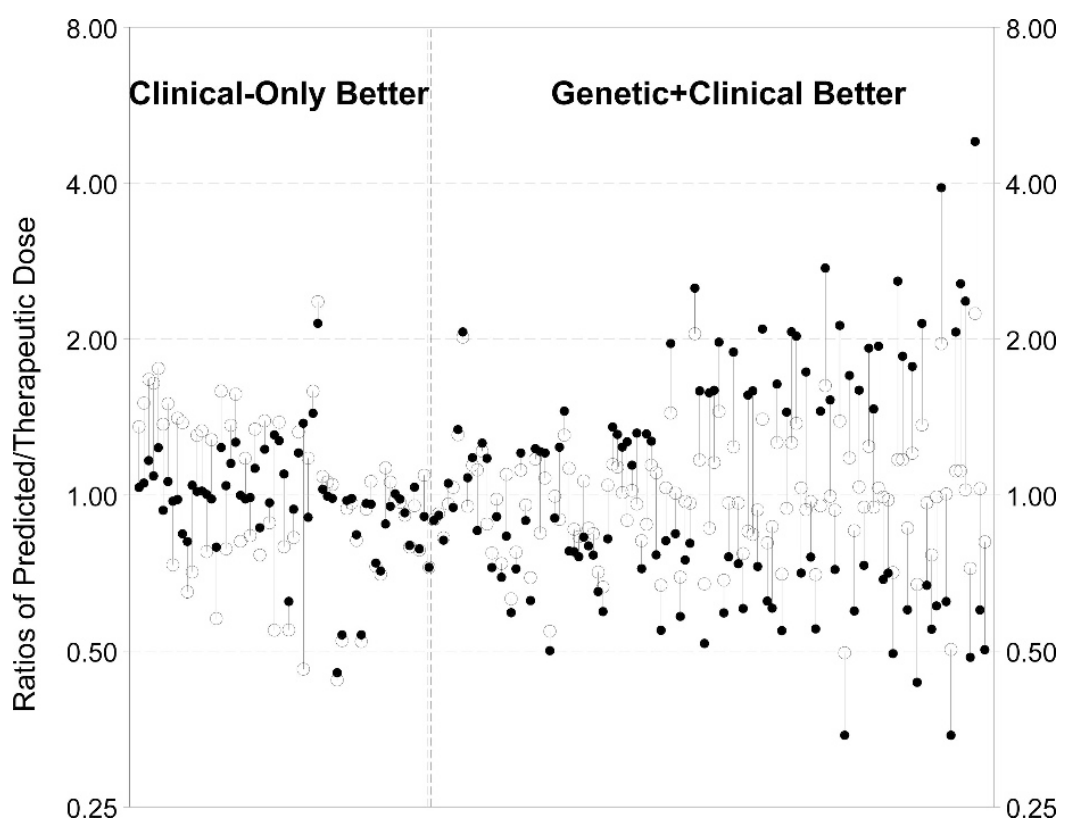

Fig. 3. Prediction by model. Model predictions relative to therapeutic observed dose are shown for both the clinical-only $(\bullet)$ and genetic + clinical $(\bigcirc)$ models. Results are scaled, such that perfect prediction of dose results in the value 1 . Results for the same subject are connected with a vertical line. Subjects are sorted by model difference, with 61 subjects where the clinical-only model gave the best prediction to the left of the vertical reference line and 115 subjects where the genetic + clinical model gave the best prediction to the right. Prediction errors were significantly smaller on average when the model included genetics $(P<0.0001)$.

\section{Time to INR $>4$}

When INR exceeds 4.0, the risk for intracerebral hemorrhage begins to exponentially increase with increase in INR. ${ }^{3}$ During the trial, INR exceeded 4.0 in 39 subjects ( $35 \%$ of 112 subjects with data) in the clinical-only arm and in 43 subjects $(38 \%$ of 113 subjects with data) in the genetic + clinical arm. The study arms were very similar with respect to the time to INR $>4.0$ $(P=0.94)$.

\section{Adverse events}

In total, 16 adverse events (eight in each arm) that met DSMB criteria for review were observed in the trial (Table 7), which includes five deaths (three cancer, one central nervous system, and one cardiac), seven hemorrhagic, and four thromboembolic events. None of the deaths were deemed study related.

All thromboembolic and hemorrhagic health events identified during the trial, whether or not they met the DSMB criteria

Table 7 Frequency of adverse events that met Data Safety Monitoring Board criteria

\begin{tabular}{lccc}
\hline & \multicolumn{3}{c}{ Randomized arm } \\
\cline { 2 - 4 } & Clinical only & Genetic + clinical & Combined \\
\hline Death during trial & 3 & 2 & 5 \\
Hemorrhagic event & 4 & 3 & 7 \\
Thromboembolic event & 1 & 3 & 4 \\
Combined & 8 & 8 & 16 \\
\hline
\end{tabular}

for review, are summarized by their severity in Table 8 . A total of 100 events (six life threatening and one fatal) in 61 subjects were observed in the clinical-only arm, whereas 112 events (two life threatening) in 54 subjects were observed in the genetic + clinical arm. Minor events (little or no clinical significance) represented $85 \%$ of the events observed.

\section{DISCUSSION}

Despite the existence of several algorithms that predict stable warfarin dose, these algorithms have not been incorporated into clinical practice because a direct patient benefit associated with genetic-based warfarin dosing has not been demonstrated. Although there is no current requirement to obtain genetic information before warfarin initiation, the Coumadin ${ }^{\circledR}$ package insert provides a range of stable maintenance doses based on combinations of $C Y P 2 C 9$ and $V K O R C 1$ gene variants and recommends, based on Food and Drug Administration guidelines, that these ranges are considered in choosing the initial dose.

Our randomized trial of genetic-based warfarin dosing used an algorithm that incorporated genotype data for $V K O R C 1$, $C Y P 2 C 9$, and $C Y P 4 F 2$, as systematic association of polymorphisms in these genes with warfarin dose has been validated, and these polymorphisms are believed to be the major genetic contributors to stable therapeutic dose of warfarin. The algorithms used for warfarin dose adjustments during warfarin initiation were those used by Marshfield Clinic's ACS. The use of these guidelines was previously shown to result in high-quality patient care, with estimated time in range $>70 \%$ for patients in maintenance phase. ${ }^{19}$

In general, patients who were dosed based on both genetic and clinical data received a starting warfarin dose that was 
Table 8 All hemorrhagic and thromboembolic events (whether or not they met Data Safety Monitoring Board criteria for review)

\begin{tabular}{|c|c|c|c|c|c|}
\hline & \multicolumn{5}{|c|}{ Frequency by severity $^{a}$} \\
\hline & Minor & Significant & $\begin{array}{c}\text { Life } \\
\text { threatening }\end{array}$ & Fatal & All \\
\hline \multicolumn{6}{|l|}{ Thromboembolic $^{b}$} \\
\hline Clinical only & 0 & 1 & 5 & 1 & 7 \\
\hline Genetic + clinical & 0 & 3 & 0 & 0 & 3 \\
\hline \multicolumn{6}{|l|}{ Hemorrhagic $^{c}$} \\
\hline Clinical only & 83 & 9 & 1 & 0 & 93 \\
\hline Genetic + clinical & 97 & 10 & 2 & 0 & 109 \\
\hline \multicolumn{6}{|l|}{ All } \\
\hline Clinical only & 83 & 10 & 6 & 1 & 100 \\
\hline Genetic + clinical & 97 & 13 & 2 & 0 & 112 \\
\hline \multicolumn{6}{|c|}{$\begin{array}{l}{ }^{a} \text { May include multiple instances per subject. } \\
\text { bathromboembolic classifications are as follows: minor-events have no clinical } \\
\text { significance or health impacts; significant-events require physician evaluation; } \\
\text { major-event requires hospitalization; life threatening - event causes irreversible } \\
\text { damage, requires an emergency procedure, or requires admission to an intensive } \\
\text { care unit; and fatal-event contributes directly to death. } \\
{ }^{c} \text { Hemorrhagic classifications are as follows: minor bleeding-bleeding with little } \\
\text { or no clinical significance that does not require any referrals to primary care } \\
\text { physician or any additional visits to primary care physician; significant bleeding- } \\
\text { bleeding that requires evaluation by primary care physicians or referral to primary } \\
\text { care physicians or associated with a decrease in hematocrit greater than } 3 \% \text { or a } \\
\text { decrease in the hemoglobin by a level of more than } 1.2 \mathrm{mg} / \mathrm{dL} \text {; major bleeding } \\
\text { event requires hospitalization and/or transfusion of at least } 2 \text { units of blood; } \\
\text { life-threatening bleeding-bleeding that has led to cardiopulmonary arrest, sur- } \\
\text { gical or angiographic, intervention, or irreversible sequelae; and fatal bleeding- } \\
\text { death is a direct result of the bleeding. }\end{array}$} \\
\hline
\end{tabular}

closer to their subsequent therapeutic dose. Our study demonstrated that an algorithm including genetic information was clearly superior at estimating therapeutic warfarin dose than an algorithm that did not consider genetic information. Compared with the standard $5 \mathrm{mg}$ /day dose, both algorithms demonstrated improved capacity to predict therapeutic warfarin dose.

Our study further demonstrated that better prediction of therapeutic dose does not necessarily result in improvements in traditional anticoagulant measures of efficacy, such as time in therapeutic range or time to stable INR. Our study reflects real-world warfarin management practices where some patients have difficulty complying with drug prescriptions and where patient management is based on INR measurements. Accordingly, our results mirror the complexities of patient management both at drug initiation and in subsequent dose adjustments early in therapy. The complexities introduced by the latter are problematic in evaluating the true impact of genetic testing because of variability in the dose-adjustment process. The trial relied primarily on hospital patients, with $80 \%$ of the patients hospitalized at the time of enrollment. The availability of more INR data in hospitalized patients may have contributed to more frequent dose adjustments than would have occurred in outpatient settings. Thus, obtaining INR measurements more than twice weekly during initiation of therapy may have created a tendency for frequent dose adjustment and consequently, "over management" of warfarin dosing. This potential effect is difficult to sort out from changes in patient condition and/or comorbidities and illness severity that may play a more prominent role among hospitalized versus outpatients. We note in this regard that of the 48 study subjects who experienced a dose change on Days 1, 2, or 3 following their initiation day, $47(98 \%)$ were in the hospital, and 32 subjects $(67 \%)$ had dose changes associated with rapidly increasing INRs (27 subjects) or low-INR response (five subjects). There were no evident randomized arm effects; 25 of 48 subjects were in the clinicalonly arm and 23 of 48 subjects in the genotype arm.

As patients were initiated on their estimated stable therapeutic dose, a related issue may have been additional uncertainty in the dose-adjustment process resulting from initiating patients on less common warfarin doses. The trial introduced initial dose recommendations that often differed from the common initiation dose of $5 \mathrm{mg} /$ day. Study nurses, including many with experience in Marshfield Clinic's ACS, may have been sensitive to these unusual initiation doses and, therefore, more likely to change dose early in the initiation process.

As in all trials, a potential limitation of our study was enrollment bias. Some physicians chose to manage otherwise eligible patients outside of the trial, and it is possible that these patients may have been systematically different from those enrolled. We do not believe that prospective participant decisions to decline enrollment presented a concern, as only $8 \%$ (20/250) approached for participation declined. Enrollment was slower than anticipated and was terminated at $88.5 \%$ of our target, reducing statistical power. However, power analysis at the time of termination determined that even continuing to full enrollment would likely not result in significant differences with respect to clinical outcomes such as time in therapeutic range.

This trial had many strengths of design including randomization of subjects before genotyping and blinding of the patients, nurses, and physicians to genotype. Ninety-two percent of trial-eligible subjects were enrolled into the trial, and primary outcome data were available for $98 \%$ of subjects for time in therapeutic range through Day 14 and $77 \%$ of subjects for prediction error assessment. The unavailability of data was similar in the two arms.

Other recent studies have investigated the utility of geneticbased warfarin dosing, and some have demonstrated a benefit of genetic-based warfarin dosing on time in therapeutic INR range and bleeding events. Caraco et al. ${ }^{27}$ demonstrated that patients on a genotype-guided initiation protocol attained their first therapeutic INR faster than controls. They also showed a significant improvement in time to stable anticoagulation, time within therapeutic range, and fewer minor bleeding events. However, they excluded in their analyses approximately one third of enrolled subjects for a number of reasons, including warfarin discontinuation, protocol violations, and study withdrawal, making it difficult to extrapolate their results to clinical management of a general warfarin patient population. Anderson et al. ${ }^{28}$ showed that genotype-guided dosing predicted stable dose more accurately than the prevailing institutional standard warfarin dosing practices. However, as in our study, their primary endpoint (percent INRs out of range) did not differ significantly between study arms.

\section{Summary interpretation of the results}

Although our study could not demonstrate that genotype-based initial warfarin dosing was superior to clinical-based dosing with respect to time in therapeutic range through the first 14 days of therapy, there was strong evidence that genotype-based dosing resulted in subjects starting warfarin on doses much closer to their therapeutic dose compared with clinically dosed subjects. Moreover, in both study arms, starting warfarin doses were closer to 
their actual therapeutic doses than they would have been if they had been initiated on a fixed $5 \mathrm{mg} /$ day regimen.

\section{ACKNOWLEDGMENTS}

Supported by the Agency for HealthCare Research and Quality (AHRQ) (1RO1HS016335-01) and by Third Wave Technologies. Supplemental funding was received from Osmetech Molecular Diagnostics. The authors are grateful to the physicians and medical staff of Marshfield Clinic who recommended patients for the trial and to Joseph Mazza, Apollo Musana, and Humberto Vidaillet for helpful suggestions. The authors thank Autumn Deedon, Sandy Strey, Debra Kempf, Lori Fellenz, Amy Vieth, Kari Lindekugel, Kathryn Smrz, Lisa Koepp, Patricia Myszka, Melissa Mikelson, and Kai Qi Zhang for their assistance with patient management during the trial. They also thank David DeMets, Justin Starren, Richard Dart, Adedayo Onitilo, and Patricia Joy Turner for participation on the DSMB. They thank Marie Fleisner of the Marshfield Clinic Research Foundation's Office of Scientific Writing and Publication for editorial assistance in the preparation of this article.

Authorship contribution: M.D.C. was the principal investigator on the study. M.D.C., S.H.Y., J.R.S., I.E.G., R.L.B., and J.K.B. designed the research. M.D.C. and S.H.Y. managed care of the patients in the trial. C.M.R. collected and managed the data. R.L.B. performed statistical analysis of the data. J.K.B., J.R.S., and I.E.G. wrote the first draft of the article. M.D.C., S.H.Y., J.R.S., I.E.G., R.L.B., and J.K.B. provided critical review and revision of the article.

\section{REFERENCES}

1. Horton JD, Bushwick BM. Warfarin therapy: evolving strategies in anticoagulation. Am Fam Physician 1999;59:635-646.

2. Wysowski DK, Nourjah P, Swartz L. Bleeding complications with warfarin use. Arch Intern Med 2007;167:1414-1419.

3. Hylek EM, Singer DE. Risk factors for intracranial hemorrhage in outpatients taking warfarin. Ann Intern Med 1994;120:897-902.

4. Glurich I, Burmester JK, Caldwell MD. Understanding the pharmacogenetic approach to warfarin dosing. Heart Fail Rev 2010;15:239-248.

5. Caldwell MD, Berg RL, Zhang KQ, et al. Evaluation of genetic factors for warfarin dose prediction. Clin Med Res 2007;5:8-16.

6. Limdi NA, Veenstra DL. Warfarin pharmacogenetics. Pharmacotherapy 2008;28:1084-1097.

7. Voora D, McLeod HL, Eby C, Gage BF. The pharmacogenetics of coumarin therapy. Pharmacogenomics 2005;6:503-513.

8. Aithal GP, Day CP, Kesteven PJ, Daly AK. Association of polymorphisms in the cytochrome P450 CYP2C9 with warfarin dose requirement and risk of bleeding complications. Lancet 1999;353:717-719.

9. Rieder MJ, Reiner AP, Gage BF, et al Effect of VKORC1 haplotypes on transcriptional regulation and warfarin dose. N Engl J Med 2005;352:2285-2293.

10. Caldwell MD, Awad T, Johnson JA, et al. CYP4F2 genetic variant alters required warfarin dose. Blood 2008;111:4106-4112.

11. McDonald MG, Rieder MJ, Nakano M, Hsia CK, Rettie AE. CYP4F2 is a vitamin K1 oxidase: an explanation for altered warfarin dose in carriers of the V433M variant. Mol Pharmacol 2009;75:1337-1346.

12. International Warfarin Pharmacogenetics Consortium, Klein TE, Altman $\mathrm{RB}$, et al. Estimation of warfarin dose with clinical and pharmacogenetic data. $N$ Engl J Med 2009;360:753-764.

13. Daly AK. Pharmacogenomics of anticoagulants: steps toward personal dosage. Genome Med 2009;1:10.

14. Hillman MA, Wilke RA, Yale SH, et al. A prospective, randomized pilot trial of model-based warfarin dose inititation using CYP2C9 genotype and clinical data. Clin Med Res 2005;3:137-145.

15. Ferder NS, Eby CS, Deych E, et al. Ability of VKORC1 and CYP2C9 to predict therapeutic warfarin dose during the initial weeks of therapy. $J$ Thromb Haemost 2010;8:95-100

16. King CR, Porche-Sorbet RM, Gage BF, et al. Performance of commercial platforms for rapid genotyping of polymorphisms affecting warfarin dose. Am J Clin Pathol 2008;129:876-883.

17. Langley MR, Booker JK, Evans JP, McLeod HL, Weck KE. Validation of clinical testing for warfarin sensitivity: comparison of CYP2C9-VKORC1 genotyping assays and warfarin-dosing algorithms. J Mol Diagn 2009;11: $216-225$.

18. Hillman MA, Wilke RA, Caldwell MD, Berg RL, Glurich I, Burmester JK. Relative impact of covariates in prescribing warfarin according to CYP2C9 genotype. Pharmacogenetics 2004;14:539-547.

19. Hillman M. Testimony before the Subcommittee on Health of the House Committee on Ways and Means, Hearing on Promoting Disease Management in Medicare, 2002. Washington, DC: U.S. House of Representatives. Available at: http://waysandmeans.house.gov/Legacy/health/107cong/4-1602/4-16hill.htm. Accessed April 16, 2002.

20. Ansell J, Hirsh J, Hylek E, Jacobson A, Crowther M, Palareti G. Pharmacology and management of the vitamin K antagonists: American College of Chest Physicians Evidence-Based Clinical Practice Guidelines (8th Edition). Chest 2008;133(suppl 6):160S-198S.

21. Rosendaal FR, Cannegieter SC, van der Meer FJ, Briët E. A method to determine the optimal intensity of oral anticoagulant therapy. Thromb Haemost 1993;69:236-239.

22. Hylek EM, Skates SJ, Sheehan MA, Singer DE. An analysis of the lowest effective intensity of prophylactic anticoagulation for patients with nonrheumatic atrial fibrillation. $N$ Engl J Med 1996;335:540-546.

23. O'Brien PC, Fleming TR. A multiple testing procedure for clinical trials. Biometrics 1979;35:549-556.

24. Box GE, Cox DR. An analysis of transformations. J Roy Stat Soc Ser B 1964;26:211-252.

25. Kaplan EL, Meier P. Nonparametric estimation from incomplete observations. J Am Statist Assn 1958;53:457-481.

26. Cox DR, Oakes D. Analysis of survival data. London, UK: Chapman and Hall, 1984.

27. Caraco Y, Blotnick S, Muszkat M. CYP2C9 genotype-guided warfarin prescribing enhances the efficacy and safety of anticoagulation: a prospective randomized controlled study. Clin Pharmacol Ther 2008;83:460-470.

28. Anderson JL, Horne BD, Stevens SM, et al. Randomized trial of genotypeguided versus standard warfarin dosing in patients initiating oral anticoagulation. Circulation 2007;116:2563-2570. 